MATEC Web of Conferences 11,01043 (2014)

DOI: $10.1051 /$ matecconf $/ 20141101043$

(C) Owned by the authors, published by EDP Sciences, 2014

\title{
Behavior of limestone filler cement mortars exposed to magnesium sulfate attack
}

\section{Comportement des mortiers à base de fillers calcaires dans une solution de sulfate de magnésium}

\author{
Y. Senhadji ${ }^{1,2}$, M. Mouli ${ }^{2}$, G. Escadeillas ${ }^{3}$, A. Khelafi, ${ }^{2,4}$, A. S. Bennosman ${ }^{5}$, R. Chihaoui ${ }^{2,4}$ \\ ${ }^{1}$ Faculté des sciences et de technologie, Université de MASCARA, Mascara 29000, Algérie \\ ${ }^{2}$ Laboratoire Lab-Mat ENSET, Département de génie civil, Oran, Algérie \\ ${ }^{3}$ Laboratoire LMDC, INSA /UPS de Toulouse : 135, Avenue de Rangueil - Toulouse, France \\ ${ }^{4}$ Laboratoire LMST/UST, Faculté d'Architecture et de Génie Civil, Med Boudiaf d'Oran, Algérie \\ ${ }^{5}$ Faculté des sciences, Laboratoire de chimie des polymères, Université d'Oran Es-Sénia, Algérie
}

\begin{abstract}
In the cement production industry, looking for a less expensive binder using industrial waste and natural resources has become a major concern for the deficit level in the manufacture of Portland cement. However, despite the technical, economic and environmental benefits brought by the use of blended cements, they are associated with disadvantages. The objective of this paper is to study the effects of the incorporation of limestone fillers on the mechanical properties and durability of mortars prepared in different combinations based on this admixture material. The durability was evaluated after immersing the specimens in a 5\% solution of magnesium sulfate for periods up to 360 days, and the penetration of chloride ions. The test results demonstrated that mortar and paste samples incorporating higher replacement levels of limestone filler were more susceptible to sulfate attack. According to microstructural analysis, such as DRX, the deterioration was significantly associated with formation of thaumasite, gypsum, and the brucite in the deteriorated parts of the specimens.
\end{abstract}

Résumé. Dans l'industrie cimentaire, la recherche d'un liant moins coûteux en utilisant des déchets industriels et des ressources naturelles est devenue une préoccupation majeure pour palier au déficit dans la fabrication du ciment Portland. Cependant, malgré les avantages techniques, économiques et écologiques rapportés par l'utilisation des ciments composés, ces derniers restent associés à des inconvénients. L'objectif de notre travail consiste en l'étude des effets de l'incorporation des fillers calcaires, sur les propriétés mécaniques et la durabilité des mortiers élaborés selon différentes combinaisons à base de cet ajout. La durabilité a été évaluée après immersion des échantillons dans une solution de $5 \%$ de sulfate de magnésium pour des périodes allant jusqu'à 360 jours, ainsi que la pénétration des ions Chlores. Les résultats des tests ont montré que les échantillons de mortier et pâte incorporant des teneurs plus élevés de remplacement de filler calcaire sont plus sensibles aux attaques des sulfates. Selon les analyses microstructurales, tel que la DRX, la détérioration a été fortement associée à la thaumasite, au gypse, et à la formation de la brucite dans la partie dégradée du matériau. 


\section{Introduction}

Les additions minérales sont de plus en plus utilisées par les producteurs de bétons. Puisqu'il s'agit généralement de sous-produits industriels, leur prise en compte dans la formulation des bétons pour le respect du dosage minimum en ciment revêt un intérêt particulier sur le plan économique (réduction du coût des matières premières) et écologique (réduction des émissions de $\mathrm{CO}_{2}$ et de la consommation de ressources non renouvelables) [1].

Les carrières gênèrent 15 millions de tonnes de sable comme un sous-produit [2]. Ce sable est rarement utilisé dans la confection des mortiers et bétons en raison de son taux élevé en fines qui varie de 8 à $30 \%$ et provoquant ainsi un problème de stockage au niveau des carrières. Plusieurs pays dans le monde tels que la France, l'Espagne, l'Angleterre, l'Argentine et la Belgique ont le même problème dans certaines régions [3], et par conséquent des programmes de recherches ont été menés pour les utilisations des sables de carrières dans la confection des mortiers et bétons. Ces études ont conduit ces pays à réviser leurs normes en particulier le taux de fines admissibles de 12 à $16 \%$ [2].

Dans cet article, nous tenterons d'apporter une contribution à la valorisation de l'utilisation du sable de concassage, après broyage, comme addition dans la fabrication du ciment Portland ordinaire. Nous présentons certains résultats relatifs au sable de concassage qui provient de la carrière de Kristel (Oran, Algérie). L'étude consiste à évaluer le comportement vis-à-vis d'une solution de sulfate de magnésium et la pénétration des ions chlores, de mortiers et bétons contenant des fillers calcaires.

\section{Matériaux et méthodes utilisés}

\section{1 Ciment}

Nous avons utilisé un ciment CEM I 42.5 de la cimenterie de Zahana. Les compositions chimiques et les caractéristiques physiques du ciment, sont données par le tableau 1.

Tableau 1. Caractéristiques chimiques et physiques du CEM I et FC

\begin{tabular}{|c|c|c|c|}
\hline \multicolumn{2}{|c|}{ Composition chimique } & Ciment CEM I 42.5 & Fillers calcaires (FC) \\
\hline \multicolumn{2}{|c|}{$\mathrm{SiO}_{2}$} & 21.35 & 7.89 \\
\hline \multicolumn{2}{|l|}{$\mathrm{Al}_{2} \mathrm{O}_{3}$} & 4.59 & 2.58 \\
\hline \multicolumn{2}{|l|}{$\mathrm{Fe}_{2} \mathrm{O} 3$} & 5.52 & 1.13 \\
\hline \multicolumn{2}{|l|}{$\mathrm{CaO}$} & 63.89 & 45.45 \\
\hline \multicolumn{2}{|l|}{$\mathrm{MgO}$} & 1.37 & 1.72 \\
\hline \multicolumn{2}{|l|}{$\mathrm{SO}_{3}$} & 2.72 & 0.21 \\
\hline \multicolumn{2}{|l|}{$\mathrm{K}_{2} \mathrm{O}$} & 0.41 & 0.95 \\
\hline \multicolumn{2}{|l|}{$\mathrm{Na}_{2} \mathrm{O}$} & 0.13 & 0.00 \\
\hline \multicolumn{2}{|l|}{$\mathrm{Cl}$} & 0.00 & 0.00 \\
\hline \multicolumn{2}{|c|}{ Perte au feu } & 0.48 & 42.48 \\
\hline \multicolumn{4}{|c|}{ Composition minéralogique } \\
\hline \multicolumn{2}{|c|}{$\mathrm{C}_{3} \mathrm{~S}$} & 47.15 & -- \\
\hline \multicolumn{2}{|l|}{$\mathrm{C}_{2} \mathrm{~S}$} & 25.69 & -- \\
\hline \multicolumn{2}{|l|}{$\mathrm{C}_{3} \mathrm{~A}$} & 2.48 & -- \\
\hline \multicolumn{2}{|l|}{$\mathrm{C}_{4} \mathrm{AF}$} & 16.70 & -- \\
\hline \multicolumn{2}{|l|}{ Calcite } & -- & 86.98 \\
\hline \multirow{2}{*}{$\begin{array}{l}\text { Temps de prise } \\
\text { (min) }\end{array}$} & Début & 103 & -- \\
\hline & Fin & 225 & $-{ }_{--}$ \\
\hline \multicolumn{2}{|c|}{ Masse volumique absolue $\left(\mathrm{kg} / \mathrm{m}^{3}\right)$} & 3100 & 2670 \\
\hline \multicolumn{2}{|c|}{ Finesse de Blaine $\left(\mathrm{cm}^{2} / \mathrm{g}\right)$} & 3500 & 3200 \\
\hline
\end{tabular}

Tableau 2. Compositions chimiques du sable de mer utilisé

\begin{tabular}{|l|c|c|c|c|c|c|c|c|c|}
\hline Eléments & $\mathrm{SiO}_{2}$ & $\mathrm{Al}_{2} \mathrm{O}_{3}$ & $\mathrm{Fe}_{2} \mathrm{O}_{3}$ & $\mathrm{CaO}$ & $\mathrm{MgO}$ & $\mathrm{SO}_{4}$ & $\mathrm{PAF}$ & $\mathrm{Cl}^{-}$ & $\mathrm{M} . \mathrm{O}$ \\
\hline Teneur (\%) & 70.88 & $\mathrm{Nul}$ & 0.64 & 15.62 & 0.14 & $\mathrm{Nul}$ & 12.91 & $\mathrm{Nul}$ & $\mathrm{Nul}$ \\
\hline
\end{tabular}

\section{2 Filler calcaire}

Le filler calcaire utilisé est un calcaire finement divisé, obtenu par broyage de roche calcaire provenant du gisement de Kristel, région de Gdyel à $30 \mathrm{Km}$ de la wilaya d'Oran. L'addition calcaire est un produit sec blanc tendre à mi-tendre essentiellement composé de carbonate $(86.64 \%)$. Le tableau 1 résume les résultats des analyses physiques et chimiques du filler calcaire.

\section{3 Sable}

Le sable utilisé est un sable de mer du type siliceux, ses caractéristiques chimiques et physiques sont consignées, respectivement dans les tableaux 2 et 3 .

\section{4 Graviers :}

Les graviers, $3 / 8$ et $8 / 15$, sont de nature calcaire provenant d'une carrière de la région d'Oran. Ils ont une dimension maximale de $15 \mathrm{~mm}$. Leurs caractéristiques chimiques principales et physiques sont représentées par les tableaux 4 et 5 . 
Tableau 3. Caractéristiques physiques du sable de mer

\begin{tabular}{|c|c|c|}
\hline \multicolumn{2}{|l|}{ Caractéristique } & Valeur \\
\hline \multicolumn{2}{|c|}{ Masse volumique absolue $\left(\mathrm{g} / \mathrm{cm}^{3}\right)$} & 2.60 \\
\hline \multicolumn{2}{|c|}{ Masse volumique apparent $\left(\mathrm{g} / \mathrm{cm}^{3}\right)$} & 1.54 \\
\hline \multirow{2}{*}{ Equivalent du sable (\%) } & Visuel & 94 \\
\hline & Piston & 90 \\
\hline \multicolumn{2}{|c|}{ Module de finesse } & 1.60 \\
\hline \multicolumn{2}{|c|}{ Coefficient de courbure : $\mathrm{C}_{\mathrm{c}}$} & 1.08 \\
\hline \multicolumn{2}{|c|}{ Coefficient d'uniformité : $\mathrm{C}_{\mathrm{u}}$. } & 1.58 \\
\hline \multicolumn{2}{|c|}{ Valeur du bleu méthylène } & 0.15 \\
\hline \multicolumn{2}{|l|}{ Nature } & Siliceuse \\
\hline
\end{tabular}

Tableau 4. Composition chimique du gravier

\begin{tabular}{|l|c|c|c|c|c|c|c|c|c|}
\hline Eléments & $\mathrm{SiO}_{2}$ & $\mathrm{Al}_{2} \mathrm{O}_{3}$ & $\mathrm{Fe}_{2} \mathrm{O}_{3}$ & $\mathrm{CaO}$ & $\mathrm{MgO}$ & $\mathrm{SO}_{4}{ }^{--}$ & PAF & $\mathrm{Cl}^{-}$ & M. O. \\
\hline Teneur (\%) & 2.65 & 0.63 & 0.39 & 51.30 & 0.89 & nul & 43.39 & Nul & Nul \\
\hline
\end{tabular}

\section{5 Formulation des mortiers}

Les mortiers utilisés sont des mortiers normalisés, conformément à la norme EN 196-1 [4]. Pour chaque série de mortiers, les mélanges ont été préparés à partir du ciment Portland CEM I 42,5 et trois combinaisons de liants obtenus suite à la substitution du ciment par différentes proportions de la pouzzolane naturelle $(0,10$, 20 et 30$) \%$ en poids du ciment. Chaque mélange permet de fabriquer simultanément trois éprouvettes cubiques (50x50x50) $\mathrm{mm}^{3}$ de mortier (tableau 5).

Tableau 5. Caractéristiques physiques des graviers

\begin{tabular}{|l|c|c|}
\hline Caractéristiques Physiques & Gravier 3/8 & Gravier 8/15 \\
\hline Masse volumique apparente $\left(\mathrm{g} / \mathrm{cm}^{3}\right)$ & 1.56 & 1.6 \\
\hline Masse volumique absolue $\left(\mathrm{g} / \mathrm{cm}^{3}\right)$ & 2,67 & 2.63 \\
\hline Absorption $(\%)$ & 0.97 & 0.84 \\
\hline Coefficient Micro Deval $(\%)$ & 22 & 24 \\
\hline Coefficient de Los Angeles $(\%)$ & 16 & 16 \\
\hline Nature & Calcaire & Calcaire \\
\hline
\end{tabular}

Tableau 6. Formulation de différents mélanges de mortiers à base de fillers calcaires

\begin{tabular}{|l|c|c|c|c|}
\hline \multirow{2}{*}{ Constituant } & \multicolumn{4}{|c|}{ Mortier pouzzolanique } \\
\cline { 2 - 5 } & FC0 & FC10 & FC20 & FC30 \\
\hline Fillers calcaires (\%) & 00 & 10 & 20 & 30 \\
\hline CEM I (\%) & 100 & 90 & 80 & 70 \\
\hline Fillers calcaires (g) & 00 & 45 & 90 & 135 \\
\hline CEM I (g) & 450 & 405 & 360 & 315 \\
\hline Sable (g) & 1350 & 1350 & 1350 & 1350 \\
\hline E/L & 0.50 & 0.50 & 0.50 & 0.50 \\
\hline
\end{tabular}

\section{6 Formulation des bétons}

Les bétons pouzzolaniques ont été confectionnés conformément à la méthode «Dreux-Gorisse » au laboratoire Lab-Mat-ENSET-Oran (tableau 7). Le rapport eau/liant est maintenu constant $(\mathrm{E} / \mathrm{L}=0,5)$. Pour le choix de cette valeur, nous avons effectué des essais de maniabilité du béton frais (essai d'affaissement-Slumptest - norme NF EN 206-1 [4]) compris entre $6 \mathrm{~cm}$ et $9 \mathrm{~cm}$. La nature et le dosage des agrégats sont maintenus constants. La composition du liant est variable en fonction du taux de substitution du ciment par les fillers calcaires $(0,10,20$ et $30 \%)$ en masse du ciment, mais le dosage global en liant est maintenu constant $=350 \mathrm{~kg} / \mathrm{m}^{3}$. 
Tableau 7. Formulation des composants des bétons pour $1 \mathrm{~m}^{3}$

\begin{tabular}{|l|c|c|c|c|}
\hline \multirow{2}{*}{ Les composants } & \multicolumn{4}{|c|}{ Différents bétons étudiés } \\
\cline { 2 - 5 } & FC0 & FC10 & FC20 & FC30 \\
\hline Fillers calcaires (\%) & 0 & 10 & 20 & 30 \\
\hline Fillers calcaires (kg) & 0 & 40 & 80 & 120 \\
\hline CEM I (\%) & 100 & 90 & 80 & 70 \\
\hline CEM I (kg) & 350 & 295 & 280 & 245 \\
\hline Gravier 8/15 (kg) & 1064 & 1064 & 1064 & 1064 \\
\hline Gravier 3/8 $(\mathrm{kg})$ & 210 & 210 & 210 & 210 \\
\hline Sable de mer $(\mathrm{kg})$ & 727 & 727 & 727 & 727 \\
\hline Eau $(\mathrm{L})$ & 180 & 180 & 180 & 180 \\
\hline E/L & 0.50 & 0.50 & 0.50 & 0.50 \\
\hline
\end{tabular}

\section{Procédure expérimentale}

\subsection{Résistance à la compression}

Après le coulage, les spécimens ont été couverts de film plastique et stockés dans l'environnement du laboratoire. Après 24 heures, les échantillons ont été démoulés et conservés dans l'eau saturée de chaux dans des bacs plastiques à une température $\left(20 \pm 2^{\circ} \mathrm{C}\right)$ jusqu'à l'âge de l'essai d'écrasement (28, 150, 240 et 360 jours).

\subsection{Attaque des mortiers par du $\mathrm{MgSO}_{4}$}

Dans le but d'accélérer le mécanisme de la dégradation des éprouvettes de mortier sous l'action de sulfates, nous avons utilisé une concentration élevée de sulfate $(5 \%$ $\left.\mathrm{MgSO}_{4}\right)$. La solution sulfatique est préparée à partir d'eau déminéralisée, à laquelle est ajouté $50 \mathrm{~g}$ de poudre pure de sulfate pour obtenir une concentration en ions sulfates de $5 \%$. La solution est homogénéisée par agitation jusqu'à dissolution complète du sulfate.

L'évolution de l'effet du milieu sulfatique sur les échantillons de mortiers confectionnés avec et sans fillers calcaires a été effectuée en la résistance à la compression, après 28, 150, 240 et 360 jours. Les solutions sulfatiques sont renouvelées chaque mois, dans le but de renouveler la quantité initiale d'ions $\mathrm{SO}_{4}{ }^{2-}$ consommée suite à l'interaction avec les matériaux cimentaires.

\subsection{L'examen visuel}

L'examen visuel périodique des échantillons en contact avec les solutions de sulfate de magnésium, avant de procéder aux essais de résistance à la compression, est un autre moyen pour évaluer la détérioration des propriétés physiques et les dégradations de l'aspect extérieur des éprouvettes et peut nous fournir des informations intéressantes, dans le cadre d'une approche comparative.

\subsection{Résistance à la pénétration d'ions chlores (ASTM C1202) [5]}

Pour les essais de pénétration d'ions chlores, nous avons utilisé des éprouvettes cylindriques $(11 \times 22) \mathrm{cm}^{2}$, ces éprouvettes sont découpées en dimensions $(110 \mathrm{~mm}$ de diamètre et $50 \mathrm{~mm}$ d'épaisseur) préparées au laboratoire Lab-Mat de génie civil de l'E.N.P d'Oran. Les mortiers ont été confectionnés conformément aux prescriptions de la norme EN 196-1 [4].

Après une période de cure de 28 jours, dans une solution saturée en chaux, La surface cylindrique de l'éprouvette est enduite d'une couche de résine époxydique étanche pour rendre les facettes imperméables, ainsi la pénétration des ions chlorure ne se fait que dans un seul sens selon la face, c.-à-d. une pénétration unidirectionnelle (figure 1). La perméabilité a été déterminée selon la norme ASTM.C-1202 [5].

L'essai consiste à mesurer l'intensité du courant engendré par une différence de potentielle égale à 60 volts maintenue constante pendant 6 heures au moyen d'électrodes en acier inoxydable entre les deux extrémités d'un échantillon de béton. L'une des faces de l'éprouvette est au contact d'une solution de $\mathrm{NaCl}$ dosé à $30 \mathrm{~g} / \mathrm{L}$ (compartiment amont : cathode), et l'autre face est au contact d'une solution de $\mathrm{NaOH}$. $0,3 \mathrm{~N}$ soit $12 \mathrm{~g} / \mathrm{L}$ (compartiment aval : anode). Les essais ont été réalisés à l'âge de 28 et 365 jours.

\section{Résultats et commentaires}

Dans cette partie, nous présentons les résultats de l'effet des fillers calcaires sur les résistances à la compression à $28,150,240$ et 360 jours, ainsi que sur la durabilité. Le calcaire est une matière première principale de l'industrie du ciment et elle est en quantités abondantes dans le nord de notre pays.

\subsection{Résistance à la compression}

D'après les figures 1-4, l'incorporation, en pourcentage élevé, des fillers provenant de la carrière de Kristel (Oran) réduit considérablement la résistance à la compression à long terme, pour les taux de substitution élevés, par rapport au ciment témoin. Nous observons une chute de résistance à la compression de 2, 10 et $21 \%$ 
pour les mortiers contenant respectivement 10,20 et 30 $\%$ de fillers calcaires par rapport à celle du mortier du ciment de référence après 360 jours. Cette addition ne possède aucune activité hydraulique en présence d'un ciment car elle est chimiquement inerte.

D'autre part, la densification de la structure par l'effet filler n'est pas forcement améliorée car le ciment est à la base très fin. Des résultats similaires avaient été observés dans des travaux antérieurs [6] et avaient été attribués au caractère inerte des fillers calcaires.

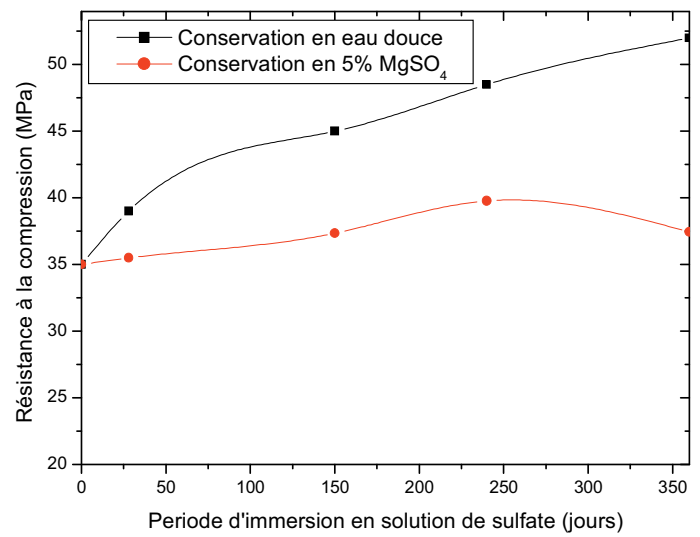

Fig. 1. Evolution de la résistance du FC0 conservé en eau douce et une solution de $5 \% \mathrm{MgSO}_{4}$

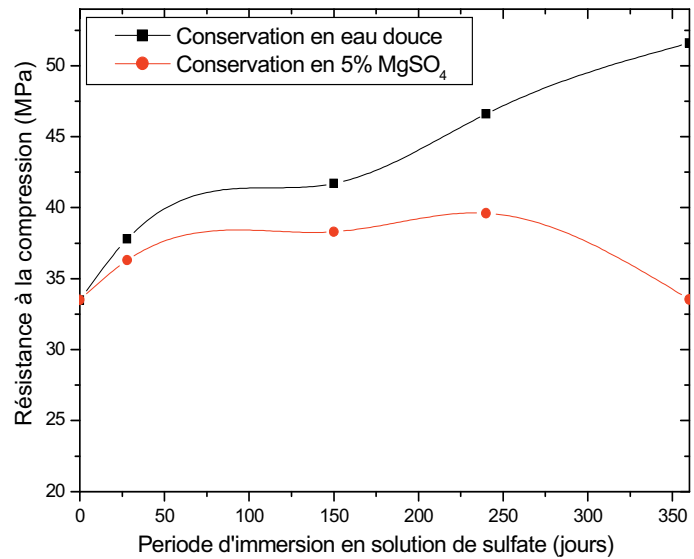

Fig. 2. Evolution de la résistance du FC10 conservé en eau douce et une solution de $5 \% \mathrm{MgSO}_{4}$

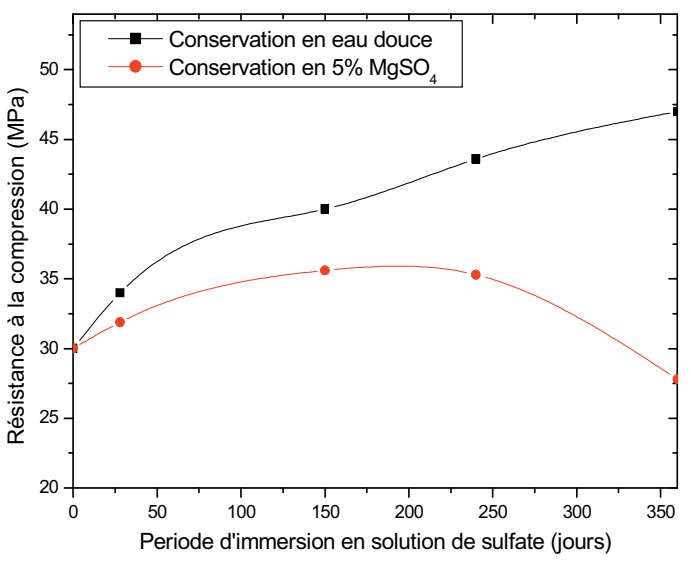

Fig. 3. Evolution de la résistance du FC20 conservé en eau douce et une solution de $5 \% \mathrm{MgSO}_{4}$

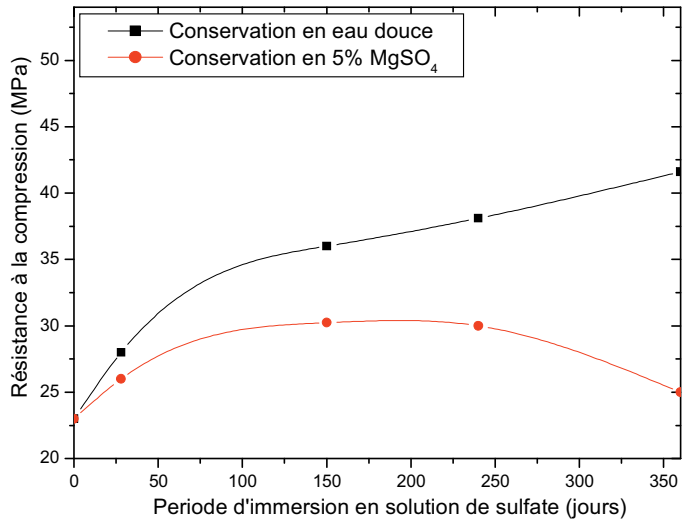

Fig. 4. Evolution de la résistance du FC30 conservé en eau douce et une solution de $5 \% \mathrm{MgSO}_{4}$

\subsection{Attaque dans une solution de sulfate de magnésium}

La figure 1 montre l'évolution de résistance des spécimens mortiers FC0 immergé dans une solution de sulfate de magnésium et stocké à $20^{\circ} \mathrm{C}$. Nous constatons que la résistance à la compression diminue avec la durée d'exposition. Le même constat peut être observé sur les mortiers FC10, FC20 et FC30 (figures 2-4). La réduction de la résistance à la compression des mortiers à base de fillers calcaires est très importante par rapport à celle des mortiers de référence $(\mathrm{FC} 0)$.

Pour l'ensemble des mélanges, l'effet du milieu sulfate sur l'évolution de la résistance à la compression est très faible durant les premières semaines d'immersion dans la solution de $5 \% \quad \mathrm{MgSO}_{4}$. Parallèlement à la dissolution de la portlandite, nous pouvons supposer une pénétration des ions sulfate dans le béton [7]. Mais cette pénétration des ions sulfate et leurs réactions éventuelles avec les produits d'hydratation ne semblent pas induire de déformation globale de l'éprouvette. 
Ce phénomène est attribué aux remplissages et la densification des pores et des micropores par des produits expansifs résultants de la réaction entre les produits d'hydratation et la solution sulfate. En effet, la réduction de la résistance à la compression est 8, 4, 4, 6\% pour les mortiers FC0, FC10, FC20 et FC30 respectivement, après 28 jours d'immersion, ce qui ne représente pas une altération significatif.

En plus, la figure 6 montre clairement qu'après 360 jours de conservation des échantillons à base de fillers calcaires, la réduction de la résistance à la compression atteint $28,35,40$ et $44 \%$ pour les mortiers FC0, FC10, FC20 et FC30 respectivement. La perte de résistance à la compression augmente lorsque le taux de substitution du filler calcaire augmente. Ces résultats prouvent l'effet négatif des FC sur l'évolution de la résistance à la compression des mortiers soumis aux attaques du sulfate de magnésium, sont en accord avec les résultats rapportés par plusieurs études [8, 9]. De nombreux chercheurs [1013] ont indiqué que la détérioration par le sulfate de magnésium de la matrice de ciment incorporant des FC, est associée à la formation des produits expansifs tels que la thaumasite, l'ettringite, le gypse, M-S-H, et la brucite. Torres et al. [12] ont rapporté que la thaumasite se forme dans les mortiers de ciment contenant $5 \%$ de fillers calcaires, ainsi que pour un remplacement plus élevé de FC $(15 \%-30 \%)$, en particulier lorsque ces mortiers ont été exposés à une solution de sulfate de magnésium et conservés une température basse $(5 \circ \mathrm{C})$.

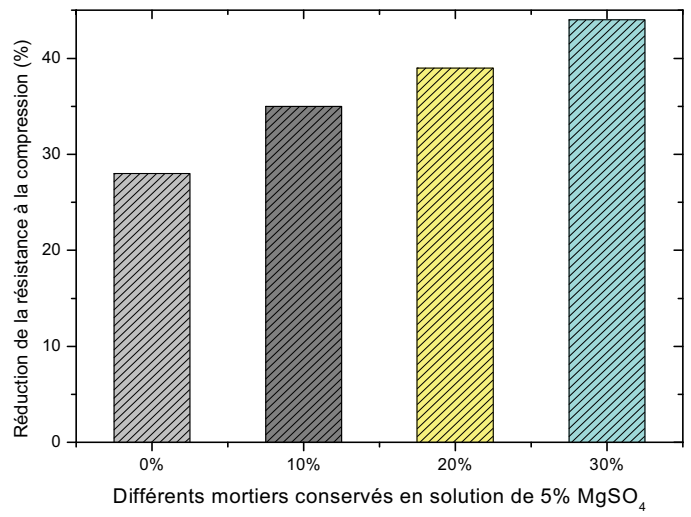

Fig. 5. Réduction de la résistance à la compression après 360 jours de conservation en $5 \% \mathrm{MgSO}_{4}$

\subsection{Examen visuel}

Des photos ont été prises pour évaluer, périodiquement, les signes de détérioration extérieure des éprouvettes de mortier tels que l'altération, la fissuration et le ramollissement après leurs conservations dans la solution de $5 \% \mathrm{MgSO}_{4}$. Les figures 6 et 7 montrent l'état des cubes $5 \times 5 \times 5 \mathrm{~cm}^{3}$ des différents mortiers à l'âge de 150 et 360 jours, respectivement.

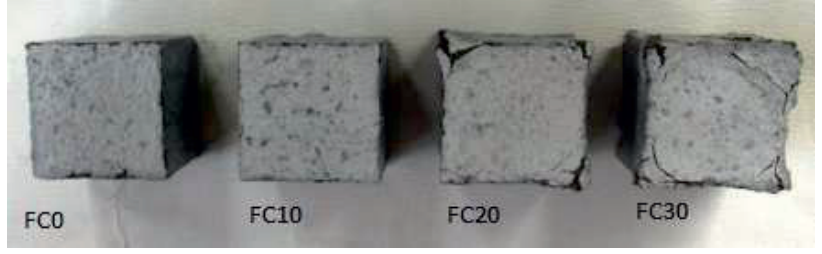

Fig. 6. Etats des échantillons après 150 jours dans $5 \% \mathrm{MgSO}_{4}$

Visuellement, nous distinguons clairement l'état des différents échantillons après 150 jours d'immersion dans la solution de sulfate de magnésium. Les mortiers à base des fillers calcaires ont perdu considérablement leur forme cubique (déformation des coins et des facettes), où l'acide a décapé la pâte de ciment. Les mortiers FC20 et FC30 ont perdu remarquablement leurs formes, principalement sur les coins et sur la surface extérieure.

La figure 7 montre moins de dégradation pour les mortiers CEM I par rapport au mortier à base de fillers calcaires. A l'œil nu, nous constatons que le volume des échantillons FC10, FC20 et FC30 est inférieur à celui de l'échantillon de référence ( $\mathrm{FC} 0)$. La dégradation, à long terme, est conséquente au point de pouvoir être suivie visuellement.

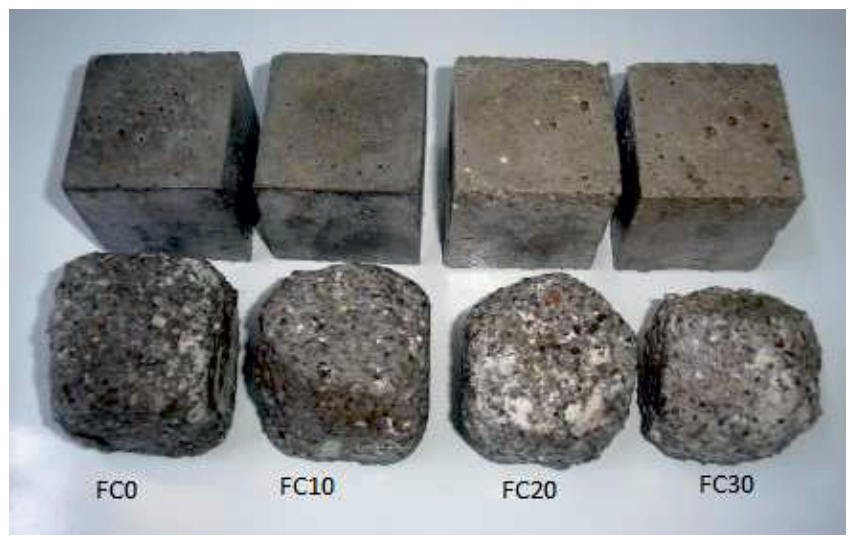

Fig. 7. Etats des échantillons après 360 jours dans: $1^{\text {ère }}$ rangée (eau douce); $2^{\text {ème }}$ rangée $\left(5 \%\right.$ de $\left.\mathrm{MgSO}_{4}\right)$

\subsection{Effet des FC sur la pénétration des chlorures (ASTM C1202-12)}

La dégradation des ouvrages en béton armé exposés aux milieux marins ou aux sels de déverglaçage est en grande partie liée au transfert des ions chlore à travers le matériau. En effet, les chlorures sont un catalyseur de la dépassivation de l'acier; première étape vers la corrosion des armatures. La pénétration des chlorures dans un environnement naturel a lieu sous l'effet de deux mécanismes: l'absorption capillaire et la diffusion. L'absorption capillaire se produit lorsque le béton sec ou partiellement saturé est imbibé par la solution saline. La diffusion résulte d'un gradient de concentration en chlorures de la solution porale entre la surface exposée et le cœur sain, elle se produit en milieu saturé. Dans le cas de cycles d'humidification et séchage, les deux mécanismes peuvent coexister. Cela concerne, par exemple, les zones de marnage des ouvrages partiellement immergés. 
La pénétration des chlorures peut-être déterminée par des essais basés sur la diffusion pure, mais ce type d'essai demande beaucoup de temps. La méthode de la norme ASTM C 1202 [5] est une méthode qui permet de mesurer rapidement le coefficient de diffusion des ions à travers le matériau béton grâce à un champ électrique.

La figure 8 montre la quantité de charge électrique, passée pendant 6 heures, à l'âge de 28 et 360 jours, pour le béton de contrôle (FC0) et les bétons substitués de (10, 20 et 30$) \%$ de fillers calcaires (FC10, FC20 et FC30). A l'opposé des résultats de l'attaque du sulfate de magnésium, les fillers calcaires réduisent considérablement la perméabilité aux ions chlores en fonction de l'augmentation du pourcentage des FC. La réduction de la charge électrique, après 360 jours, est de $6 \%, 11 \%$ et $15 \%$ pour FC10, $\mathrm{FC} 20$ et $\mathrm{FC} 30$ respectivement par rapport au béton de contrôle. Ceci est dû au raffinement des pores des bétons à base de FC et la réduction de la conductivité électrique des bétons à base de FC. D'autre part, la morphologie du réseau poreux, des bétons à base de FC sont différentes, cela se traduirait par une porosité capillaire et des coefficients de diffusion plus faibles [6].

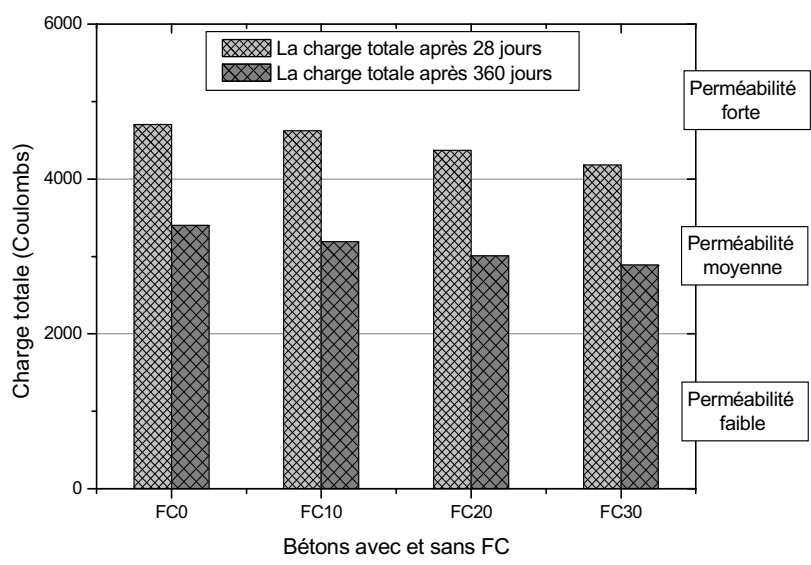

Fig. 8. Charge totale après 28 et 360 jours des différents bétons

\subsection{Etude DRX des mortiers suites à l'attaque du $\mathrm{MgSO}_{4}$}

La minéralogie des mortiers de ciment, à base de 0,10 , 20 et $30 \%$ de $\mathrm{FC}$, a été caractérisée par diffraction des rayons $\mathrm{X}$, après immersion pendant 360 jours dans la solutions magnésium. A partir de ces spectres (figure 9), nous pouvons remarquer une grande quantité de calcite (C) (avant et après attaque) due au sable utilisé pour la confection des mortiers. Ces spectres révèlent la présence d'ettringitte (Et), de thaumasite (Th), de gypse (G), de portlandite (Por), de calcite $(\mathrm{C})$, et de brucite $(\mathrm{Br})$.

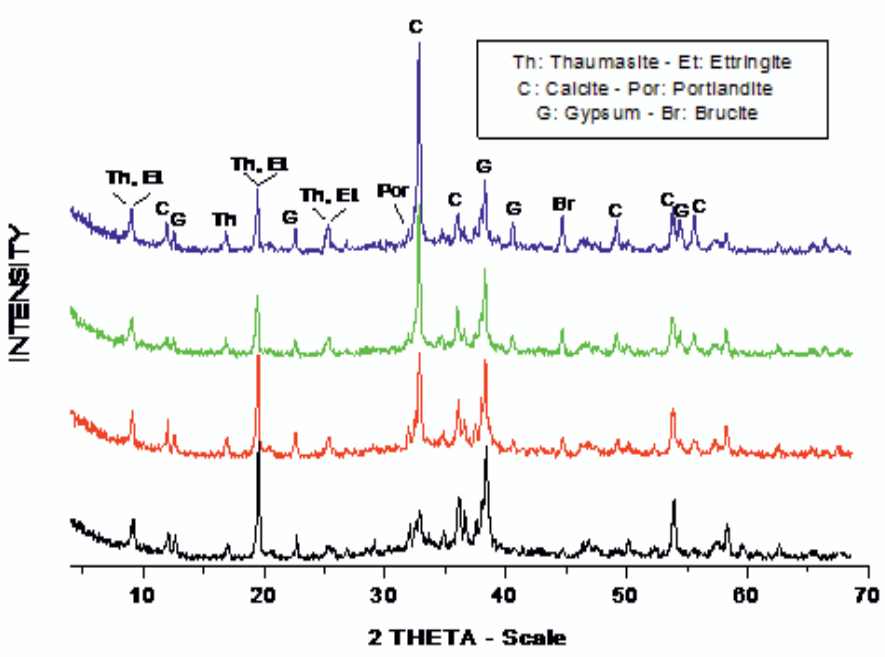

Fig. 9. Diagramme $\mathrm{DRX}$ de mortiers à base de $\mathrm{FC}$ ayant été immergée 360 jours dans $5 \%$ de $\mathrm{MgSO}_{4}$ Noir: FC0; Rouge: FC10; Vert: FC20; Bleu: FC30

L'intensité des pics du gypse est relativement plus importante, en particulier pour l'échantillon FC30, par rapport aux pics d'autres produits formés. Les pics de la portlandite, dans les spectres des échantillons FC20 et FC30 sont très faibles, $\mathrm{Le} \mathrm{Ca}(\mathrm{OH})_{2}$ a probablement réagi avec le sulfate de magnésium pour former le gypse et la portlandite.

\section{Conclusion}

Les résultats obtenus à partir de cette étude expérimentale, nous permettent de tirer les conclusions suivantes :

- La résistance à la compression du mortier à base de fillers calcaires, diminue avec l'augmentation du taux d'incorporation des FC. Le FC10 donne des caractéristiques très proches de celles du ciment CEM I .

- La dégradation des mortiers contenant des fillers calcaires est plus prononcée que le mortier témoin. Plus la teneur est élevée, plus l'échantillon est altéré.

- Les FC améliorent l'impénétrabilité des matériaux cimentaires et augmentent ses résistivités, contribuant ainsi à réduire la vitesse de corrosion. L'incorporation des FC apportent les modifications suivantes :

- Réduction da la perméabilité du matériau.

- Réduction de la taille des pores.

\section{References}

1. Skaropoulou, A, Tsivilis, S, Kakali, G, Sharp, J and Swamy, R, Construction and Building Materials, 23(6) (2009).

2. Bertrandy. R, Coquillat. G et Giordano. R, Revue recherche France septembre 93, 147-150, (1993).

3. R. Chaid, R. Jauberthie et A. Boukhaled, Lebanese Science Journal, Vol. 11(1), (2010). 
4. EN 196-1, Methods of testing cement - Part 1: determination of strength. CEN (1995).

5. Norme ASTM C1202-12: « standard method for electrical indication of concrete's ability to resist chloride ion penetration», (2012).

6. Senhadji Y., Mouli M., Khelafi H. Benosman A. S., Turkish J. Eng. Env. Sci. 34, 131 - 143, (2010).

7. Planel D. «Les effets couplés de la précipitation d'espèces secondaires sur le comportement mécanique et la dégradation chimique des bétons ». Thèse, Université de Marne-la vallée (2002).

8. Justnes, H., Cement and Concrete Composites, 25, 955-959, (2003).

9. Pipilikaki, P., Katsioti, M. Gallias, J.L., Construction and Building Materials, 23, 1042-1049, (2009).
10. Hartshorn, S.A., Swamy, R.N. Sharp, J.H., Advances in Cement Research, 13, 31-46, (2001).

11. Torres, S.M., Sharp, J.H., Swamy, R.N., Lynsdale, C.J. and Huntley, S.A., Cement and Concrete Composites, 25, 8947-8954, (2003).

12. Tsivilis, S., Kakali, G., Skaropoulou, A., Sharp, J.H., Swamy, R.N., Cement and Concrete Composites, 25, 969-976, (2003).

13. Irassar, E.F., Bonavetti, V.L., Trezza, M.A. Gonzalez, M.A., Cement and Concrete Composites, 27, 77-84, (2005). 